\title{
Application of Oral Intermittent Catheterization in Stroke Patients with Dysphagia
}

\author{
Haiyan Yang ${ }^{1}$ Yong Yu ${ }^{1,2, *}$, Zhiyong Peng ${ }^{1}$ \\ ${ }^{1}$ Department of Rehabilitation Medicine, Yuedong Hospital, Third Affiliated Hospital of Sun Yat-sen University, China. \\ ${ }^{2}$ Department of Rehabilitation Medicine, The Third Affiliated Hospital of Sun Yat-sen University, China.
}

How to cite this paper: Haiyan Yang, Yong Yu, Zhiyong Peng. (2021) Application of Oral Intermittent Catheterization in Stroke Patients with Dysphagia. International Journal of Clinical and Experimental Medicine Research, 5(1), 11-14.

DOI: 10.26855/ijcemr.2021.01.003

Received: November 19, 2020

Accepted: December 20, 2020

Published: January 4, 2021

*Corresponding author: Yong Yu, Department of Rehabilitation Medicine, Yuedong Hospital, Third Affiliated Hospital of Sun Yat-sen University, China; Department of Rehabilitation Medicine, The Third Affiliated Hospital of Sun Yat-sen University, China.

Email:27847739@qq.com

\begin{abstract}
Objective: to explore the clinical application of oral intermittent catheterization in stroke patients with dysphagia. Methods: Forty patients with dysphagia selected by the drinking water test were randomly divided into treatment group and control group. The control group used traditional indwelling gastric tube, and the treatment group used intermittent tube indwelling. The thickness of the upper arm triceps skinfold, the changes in serum albumin, and the incidence of aspiration and pneumonia were compared before hospitalization and 4 weeks after treatment. Results: After 4 weeks of treatment, the thickness of upper arm triceps skin folds and serum albumin levels in the two groups were significantly higher than before treatment $(\mathrm{P}<0.05)$, and the change in the treatment group was better than that in the control group $(\mathrm{P}<0.05)$; the treatment group was aspirated. The incidence was $5 \%$, which was lower than $20 \%$ in the control group $(\mathrm{P}>0.05)$; the incidence of pneumonia in the treatment group was $5 \%$, which was lower than $15 \%$ in the control group ( $\mathrm{P}>0.05)$, but the difference was not statistically significant. Conclusion: Intermittent catheterization can significantly improve the nutritional status of patients, prevent aspiration and pulmonary infection, and improve swallowing function. It is worthy of clinical application.
\end{abstract}

\section{Keywords}

Stroke, Intermittent Catheterization Through The Mouth, Dysphagia

\section{Introduction}

The incidence of dysphagia after stroke is high, and dysphagia is one of the common complications of stroke patients [1]. Due to impaired esophageal function, the patient cannot effectively deliver food from the mouth to the stomach, causing serious problems such as malnutrition, aspiration, and lung infections [2]. Intermittent catheterization can not only solve the intake of nutrients and water required by patients with dysphagia, but also rebuild the patient's personal image, and the swallowing function is also greatly improved [3]. The report is as follows.

\section{Materials and Methods}

\subsection{General information}

Inclusion criteria: (1) Comply with the stroke diagnostic criteria formulated by the 4th Cerebrovascular Disease Academic Conference of the Chinese Medical Association in 1995 [4] and the stroke was diagnosed by CT or MRI examination of the brain; (2) The cricopharyngeal muscle was diagnosed by the swallowing contrast examination 
Achalasia; (3) Swallowing dysfunction after clinical evaluation; (4) Clear consciousness and stable vital signs; (5) Able to cooperate with the required assessment and examination; (6) Sign informed consent.

Exclusion criteria: (1) Patients with critical illness or failure of important organs; (2) Patients with severe cognitive or mental disorders; (3) Patients with abnormal structures of the oral cavity, pharynx and esophagus in the past;

Select 40 patients with dysphagia after stroke who were admitted to our hospital from January 2019 to July 2019. Patients with dysphagia can be judged by two methods: (1) Watian drinking test: Instruct patients to drink 30mL of warm water according to their habits, and observe the frequency of drinking water and coughing. The test divides the swallowing function into 5 levels, the higher the level, the more severe the swallowing dysfunction. Level 1: Can swallow water smoothly once; Level 2: Divide more than 2 times, swallow water without coughing; Level 3: Can swallow once, but cough; Level 4: Divide 2 Swallowing water more than once, but coughing; Grade 5: Cannot swallow all, and frequent coughing. (2) Food intake-Swallowing function score: The total score is 10 points, the lower the score, the more severe the swallowing disorder. 1-3 points: Severe dysphagia, completely dependent on supplementary feeding, unable to eat by mouth; 4-6 points: Moderate dysphagia, part of the nutrition can be ingested by mouth, and part of the nutrition needs to be ingested through intravenous injection; 7-9 Points: Mild dysphagia, nutrition can be taken by mouth, but compensation and adaptation are needed; 10 points: Ingestion-swallowing function is completely normal. They were randomly divided into two groups. All cases were checked and evaluated by a rehabilitation therapist within 3 days after admission. In the control group, there were 12 males and 8 females, aged 52-78 years, with an average age of (55.75 \pm 10.61$)$ years; in the treatment group, there were 14 males and 6 females with an average age of (55.30 8.58$)$ years. The above-mentioned data of the two groups of patients were not statistically significant and comparable.

\subsection{Intervention methods}

Control group: Long-term indwelling gastric tube nasal feeding was used, that is, the gastric tube was inserted into the stomach from the nasal cavity, and the gastric tube was properly fixed to the nose. Nasal feeding was performed 4 times a day according to the patient's digestion. After the infusion, the end of the stomach tube is raised and folded, wrapped with gauze, tied tightly with a rubber band, and fixed on the patient's pillow, bed sheet or collar with a pin.

Treatment group: A silicone double-chamber balloon catheter was intermittently inserted through the mouth to the esophagus, with a depth of $18 \mathrm{~cm}-23 \mathrm{~cm}$. When inserted into the throat, the patient was asked to swallow, and $3-5 \mathrm{ml}$ water lift catheter was injected into the catheterization balloon and stayed. Where there is a sense of jam, make sure that the water bladder is stuck on the lower edge of the cricopharyngeal muscle, and place the end of the catheter in a treatment bowl filled with water. There is no continuous air bubble overflow. Instruct the patient to make a "yi" sound, and the sound is clear. Observe whether the patient has cough, slowly infuse the nasal feeding solution, and instruct the patient to swallow while injecting. The amount of tube feeding is $300-400 \mathrm{ml}$ each time, 4-6 times a day. After the perfusion is completed, a small amount of saline is injected again, the water injected into the balloon is drained, and the catheter is slowly pulled out. Instruct the patient to sit and lie down for 30 minutes.

\subsection{Observation indicators}

1. Nutritional indicators: (1) Upper arm triceps skinfold thickness (TSF): The specific measurement method is that the patient's upper arm sags naturally, and the midpoint of the left upper arm is taken, which is about $2 \mathrm{~cm}$ above the midpoint of the line from the acromion to the olecranon, Use the left thumb and index finger to pinch the skin and subcutaneous fat into folds, and measure with a skinfold thickness gauge with a pressure of $10 \mathrm{~g} / \mathrm{mm} 2$; take the average value after 3 consecutive measurements. Measure the circumference of the upper arm with a soft ruler on the same part. The standard value adopts the index recommended by FQO/WHO. The normal value is $12.5 \mathrm{~mm}$ for males and $16.5 \mathrm{~mm}$ for females. A $10 \%$ decrease is malnutrition [5]; (2) Normal serum albumin value: 35-50g/L; these indexes represent the nutritional status of the patient's fat and internal organs.

2. Complications: (1) Swallow $5 \mathrm{ml}, 10 \mathrm{ml}$, and $20 \mathrm{ml}$ of warm boiled water in a awake state in the sitting or semi-recumbent position for the aspirated patient. The interval between each dose is 30s. If coughing, breathing difficulties, swallowing occur a change in the quality of the posterior sound is a positive aspiration; (2) The criteria for a positive lung infection are as follows: the patient has symptoms of respiratory tract infection such as cough, sputum, and fever, and CT examination of the lungs confirms inflammation;

3. Serum albumin level: blood samples will be taken on the second day and the fourth week of hospitalization to test the serum albumin level; 


\subsection{Statistical analysis}

The SPSS19.0 statistical software package was used to process the data. The measurement data were expressed as mean \pm standard $(\mathrm{x} \pm \mathrm{s})$, the count data used the $\chi 2$ test, and the measurement data used two independent samples $\mathrm{t}$ test. $\mathrm{P}<0.05$ was used as the statistical difference. Learn meaning.

\section{Results}

1. Nutritional indicators: There was no statistically significant difference in the thickness of the triceps skinfold and serum albumin between the two groups of patients after treatment $(\mathrm{P}>0.05)$. After 4 weeks of treatment, the thickness of the upper arm triceps skinfold and serum albumin levels in the two groups were significantly higher than before treatment $(\mathrm{P}<0.05)$, and the change in the treatment group was better than that in the control group $(\mathrm{P}<0.05)$, see Table 1.

Table 1. Comparison of upper arm triceps skinfold thickness and serum albumin index before and after treatment

\begin{tabular}{llll}
\hline Group & Time & Upper arm triceps skinfold thickness mm & Serum albumin g/L \\
\hline \multirow{2}{*}{ Control group $(\mathrm{n}=20)$} & Before treatment & $9.32 \pm 1.33$ & $25.93 \pm 2.45$ \\
& 4 weeks after treatment & $10.3 \pm 1.53^{*}$ & $35.760 \pm 2.53^{*}$ \\
& Before treatment & $9.25 \pm 1.31$ & $26.15 \pm 2.37$ \\
Treatment group $(\mathrm{n}=20)$ & 4 weeks after treatment & $12.30 \pm 1.45^{*} \triangle$ & $40.58 \pm 2.25^{*} \triangle$ \\
\hline
\end{tabular}

Note: Compared with before treatment, ${ }^{*} \mathrm{P}<0.05$; compared with control group, $\triangle \mathrm{P}<0.05$.

2. Comparing the complications of the two groups of patients, the incidence of aspiration in the treatment group was $5 \%$, which was lower than $20 \%$ in the control group ( $P>0.05)$; the incidence of pneumonia in the treatment group was $5 \%$, which was lower than $15 \%$ in the control group $(\mathrm{P}>0.05)$, the difference was not statistically significant. See Table 2.

Table 2. Comparison of complications in the 2 groups [n (\%)]

\begin{tabular}{llll}
\hline Group & Number of cases & Incidence of aspiration & Incidence of pneumonia \\
\hline Control group & 20 & $4(20)$ & $3(15)$ \\
Treatment group & 20 & $1(5)$ & $1(5)$ \\
\hline
\end{tabular}

\section{Discussion}

Swallowing disorder after stroke is one of the common dysfunctions in patients. Pay attention to the management of dysphagia after stroke, which plays a pivotal role in improving the quality of life of stroke patients and reducing the disability rate and mortality rate [5]. Recovery of patients with swallowing dysfunction is a long and complex process. In clinical practice, long-term placement of nasogastric tubes in patients with dysphagia can cause nasopharyngeal and esophageal mucosal ulcers, bleeding, reflux esophagitis, and increase the chance of aspiration, aspiration pneumonia, and gastrointestinal infections [6]. On the one hand, the stimulation of the foreign body after the indwelling of the gastric tube increases the secretions of the respiratory tract and oral cavity; on the other hand, the indwelling of the gastric tube makes the lower esophageal sphincter relatively incompletely closed, and the indwelling of the gastric tube further weakens the swallowing reflex [7]. Intermittent oral infusion can not only play a role in compensatory eating, but also a treatment for swallowing dysfunction. This method can meet the needs of patients for water and nutrients [8]. This study showed that the thickness of the upper arm triceps skinfold and the level of serum albumin in the treatment group changed better than those in the control group after 4 weeks of treatment $(\mathrm{P}<0.05)$. The reason may be related to the fact that food enters the esophagus through the mouth through the tube to the stomach. Compared with intermittent nasal feeding, it is more in line with the physiological law of normal eating. The tube can be pulled out immediately after eating, which has little impact on rehabilitation function training and quality of life, and promotes the body the nutritional status is improved as soon as possible. In addition, patients with oral intermittent gastric tube insertion must have their own actions to swallow the gastric tube multiple times each time the gastric tube is inserted, so as to train the relevant muscles involved in swallowing during the pharyngeal period, which is beneficial to promote the oral cavity and pharyngeal cavity. It has the function of improving swallowing dysfunction. The incidence of aspiration and lung infection in the treatment group 
was lower than that in the observation group, but the difference was not statistically significant. This may be related to the number of cases and the severity of the disease.

The sample size in this study is insufficient, and there are relatively few reports of adverse events. In future studies, the sample size can be increased and patients can be followed up and followed up for a long time to discover adverse reactions and problems during intermittent catheterization operations, and provide evidence-based evidence for clinical work to ensure that patients get more comprehensive, effective, high-quality treatment and care.

In summary, this study believes that intermittent catheterization can significantly improve the nutritional status of patients, prevent aspiration and lung infection, improve swallowing function, and effectively reduce complications such as dehydration, electrolyte imbalance, weight loss, etc. It is worthy of clinical application.

\section{References}

[1] Li Bingjie, Zhang Tong, Zhao Jun. (2011). The effect of dysphagia on the nutritional status of stroke rehabilitation patients [J]. Chinese Rehabilitation Theory and Practice, 17(3): 262-264.

[2] Cao Gaofan. (2016). The application effect of intermittent tube feeding on the improvement of swallowing function in elderly stroke patients with dysphagia [J]. Chinese Continuing Medical Education, 8(17): 230-231.

[3] Dou Zulin (editor in chief). (2009). Evaluation and treatment of dysphagia [M]. Beijing: People’s Medical Publishing House, 2009: 290-291.

[4] Chinese Neuroscience, Chinese Society of Neurosurgery, Essentials of Diagnosis of Various Cerebrovascular Diseases [J]. Chinese Journal of Neurology 1996, 29(6): 379-380.

[5] Wu Xiaohua, Zhao Xining, Zhang Huahua, et al. (2016). Comparison of the nutritional effects of oral feeding and nasal feeding in patients with dysphagia [J]. Contemporary Nurses, 5(3): 105-107.

[6] Zhu Meihong, Shi Meifang, Wan Lihong, et al. (2016). Swallowing-feeding management to prevent stroke associated pneumonia in patients with dysphagia [J]. Chinese Journal of Nursing, 51(3): 294-298.

[7] Guo Fuxia, Zhong Defu, Chen Shumin, et al. (2010). The effect of the duration of semi-recumbent position after nasal feeding in stroke patients with dysphagia on aspiration [J]. Journal of Nursing, 17(8A): 66.

[8] Daxing Xizi, Sun Qiliang. (2000). Practical techniques for the rehabilitation of eating and swallowing disorders [M]. Beijing: China Science and Medicine Press, 2000: 95-98. 